\title{
Large-signal PIN diode model for ultra-fast photodetectors
}

\author{
Krozer, Viktor; Fritsche, C
}

Published in:

European Microwave Conference, 2005

Link to article, DOI:

10.1109/EUMC.2005.1610152

Publication date:

2005

Document Version

Publisher's PDF, also known as Version of record

Link back to DTU Orbit

Citation (APA):

Krozer, V., \& Fritsche, C. (2005). Large-signal PIN diode model for ultra-fast photodetectors. In European Microwave Conference, 2005 (Vol. 2). IEEE. https://doi.org/10.1109/EUMC.2005.1610152

\section{General rights}

Copyright and moral rights for the publications made accessible in the public portal are retained by the authors and/or other copyright owners and it is a condition of accessing publications that users recognise and abide by the legal requirements associated with these rights.

- Users may download and print one copy of any publication from the public portal for the purpose of private study or research.

- You may not further distribute the material or use it for any profit-making activity or commercial gain

- You may freely distribute the URL identifying the publication in the public portal

If you believe that this document breaches copyright please contact us providing details, and we will remove access to the work immediately and investigate your claim. 


\title{
Large-signal PIN diode model for ultra-fast photodetectors
}

\author{
Carsten Fritsche and Viktor Krozer \\ Technical University of Denmark, Oersted•DTU, Department of Electromagnetic Systems, \\ Oersteds Plads 348, 2800 Kgs. Lyngby, Denmark, Phone:+45-45253769, E-mail:vk@oersted.dtu.dk \\ and Center for Applied Technology and Electronics ATHENE e.V., Darmstadt, Germany
}

\begin{abstract}
A large-signal model for PIN photodetector is presented, which can be applied to ultra-fast photodetection and THz signal generation. The model takes into account the tunnelling and avalanche breakdown, which is important for avalanche photodiodes. The model is applied to ultra/fast superlattice photodiodes for $\mathrm{THz}$ signal generation. Results show that the output power at $\mathrm{THz}$ frequencies is in the order of tens of $\mu \mathrm{W}$. The embedding impedances are found to be as low as $13 \Omega$.
\end{abstract}

\section{INTRODUCTION}

A large amount of literature exists on the performance and circuit modelling of PIN diodes both for electrical and optical applications. A number of models exist for the PIN photodiode, which are in general small-signal models, including the models for travelling-wave structures [1], [2], [3], [4]. Most models do net lend themselves to implementation in a circuit simulator, because they are either based on numerical techniques [5] or are based on blackbox models extracted from small-signal measurements. Parasitics modelling of fast PIN photodiodes has been presented by Wang et al .

A PIN photodiode model suitable for circuit simulations has been presented in [6] for avalanche photodiodes and for reverse bias only. This model has been used as a basis for the development of the large-signal model for standard PIN and superlattice PIN photodiodes and has been employed for the implementation into the circuit simulator.

This paper gives details on the model and compares results from measurements with simulations. Both avalanche InGaAs photodiodes as well as GaAs PIN $\mathrm{THz}$ photodiodes are considered in the discussion. To our knowledge we demonstrate for the first time a PIN device large-signal model suitable up to $\mathrm{THz}$ frequencies implemented into a commercial CAD tool. The model is then used for the prediction of the signal output from the photodetector up to $\mathrm{THz}$ frequencies and the determination of the embedding impedances.

\section{PIN PHOTODIODE LARGE-SIGNAL MODEL}

The PIN model is based on the drift-diffusion model with a modified velocity versus field characteristic and takes into account the rate equations. The following assumptions are made, which are generally valid for high speed devices:

1) The depletion width in the $n^{+}$and $p^{+}$regions is negligible compared to that in the I-region.

2) The electric field is uniform in the I-region.

3) The electric field is negligible in the $n^{+}$and $p^{+}$ regions.
4) In real devices the I-region contains residual doping, which is used as a parameter in the model.

The model equations can be divided into those describing the optical performance and those determining the electrical performance of the device, respectively. Illumination is currently assumed from the $n^{+}$side, but can be changed to illumination from the $p^{+}$side by interchanging the parameters of the $n^{+}$-region with those of the $p^{+}$-region. A diffusion current conduction is assumed for the highly doped regions and drift conduction in the I-region. The rate equations can be given in the following form for the $n^{+}$-region:

n-region:

$$
\frac{\mathrm{d} P_{n}}{\mathrm{~d} t}=P_{G}-\frac{P_{n}}{\tau_{p}}-\frac{I_{p}}{q}
$$

i-region: for electrons

$$
\frac{\mathrm{d} N_{i}}{\mathrm{~d} t}=N_{G i}+v_{n} \zeta_{n} N_{i}+v_{p} \zeta_{p} P_{i}-\frac{N_{i}}{\tau_{n r}}-\frac{N_{i}}{\tau_{n t}}+\frac{I_{n}}{q}
$$

p-region:

$$
\frac{\mathrm{d} N_{p}}{\mathrm{~d} t}=N_{G}-\frac{N_{p}}{\tau_{n}}-\frac{I_{n}}{q}
$$

where the $P_{n}$ and $N_{p}$ are the total excess holes and electrons in the n- and p-regions, $N_{i}$ are the total excess electrons in the i-region, q the electron charge, $P_{G}$ and $N_{G}$ are electron-hole pair generation rates in the n- and p-regions by incident light, $N_{G i}\left(=P_{G i}\right)$ is the generation rate in the i-region, $\tau_{p}$ and $\tau_{n}$ are the hole and electron life-time in the $\mathrm{n}$ - and p-regions, $\tau_{n r}$ and $\tau_{p r}$ are the recombination life-time of electron and hole in the iregion, $\tau_{n t}$ and $\tau_{p t}$ are the electron and hole transit-time through the i-region, $I_{p}$ and $I_{n}$ are the hole and electron diffusion current in the $\mathrm{n}$ - and p-regions, $v_{n}$ and $v_{p}$ are the electron and hole drift velocities in the i-region, and $\zeta_{n}$ and $\zeta_{p}$ are the electron and hole impact ionization rates in the i-region. The schematic structure of a PIN is shown in fig. 1. The solution of the above equations determines the charge flow in the device and its frequency and voltage dependent performance. The solution is found by applying the according boundary conditions as outlined in [6]. The electron-hole pair generation rate is given

$$
G(x)=\Phi_{0} \alpha \exp (-\alpha x)
$$

where $\Phi_{0}$ is the incident photon flux per unit area given by $P_{i n}(1-R) / A h \nu$, where $\mathrm{R}$ is the facet reflectivity of the n-region, $h \nu$ is the photon energy and $\mathrm{A}$ is the device area, and $\alpha$ is the absorption coefficient for the individual semiconductor layer. 


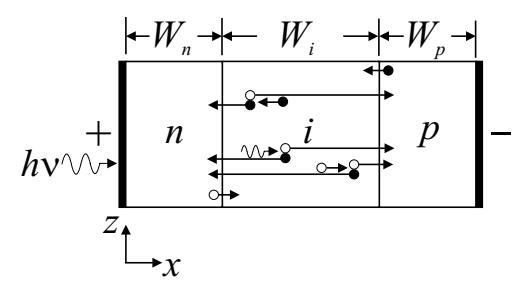

Fig. 1. Schematic illustration of carrier fbw in a PIN diode.

The proposed equivalent circuit has the structure as shown in Fig. 2. It contains an optical part and an electronic part. These two are coupled via controlled current and voltage sources. In the case of multi-section devices the equivalent circuit is being connected is series on both the electrical and optical ports. The resistor $R_{u u}$ is then employed only once in the circuit, because it represents the optical power delivered to the substrate. Adding mirrors can be taken into account by appropriate manipulation of additional absorption terms. Therefore, it is believed that the equivalent circuit is rather complete including the dominant physical phenomena. The optical

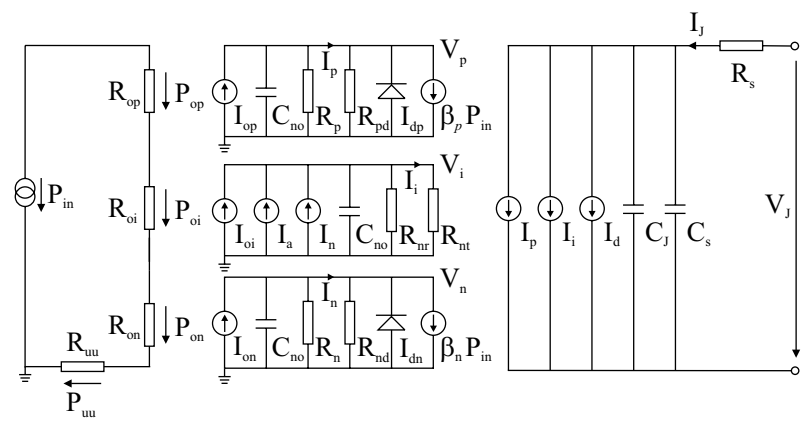

Fig. 2. Electrical and optical equivalent circuit for the PIN photodetector.

input power $P_{i n}$ is represented by the equivalent voltage source. The power $P_{o p}$ represents the amount of the power $P_{i n}$ that will be dissipated on the resistance $R_{o p}$ due to generation of electron-hole pairs in the n-layer. The power $P_{o i}$ is that part of the total power $P_{i n}$ that will be dissipated on the resistance $R_{o i}$. Photons that will be absorbed in the i-layer and generate electron-hole pairs are represented by the power $P_{o n}$ and its resistance $R_{o n}$. The photons that will not be absorbed in the APD can be represented by the amount of power $P_{u u}$ that is dissipated on the resistance $R_{u u}$.

The rate equations can be transformed to current equations by multiplying each equation with the electron charge q. In order to improve numerical accuracy, a constant $C_{n o}$ is introduced, which can be thought as a capacitance. Defining the following equivalent voltages

$$
V_{p}=\frac{q P_{n}}{C_{n o}} \quad V_{n}=\frac{q N_{p}}{C_{n o}} \quad V_{i}=\frac{q N_{i}}{C_{n o}}
$$

the rate equations (1),(3) and (??) can be written as

$$
\frac{P_{o p}}{V_{o p t}}=C_{n o} \frac{\mathrm{d} V_{p}}{\mathrm{~d} t}+\frac{V_{p}}{R_{p}}+I_{p}
$$

$$
\begin{aligned}
\frac{P_{o n}}{V_{o p t}} & =C_{n o} \frac{\mathrm{d} V_{n}}{\mathrm{~d} t}+\frac{V_{n}}{R_{n}}+I_{n} \\
\frac{P_{o i}}{V_{o p t}} & =C_{n o} \frac{\mathrm{d} V_{i}}{\mathrm{~d} t}+\frac{V_{i}}{R_{n r}}+\frac{V_{i}}{R_{n t}}-I_{a}-I_{n}
\end{aligned}
$$

where

$$
\begin{aligned}
R_{p} & =\frac{\tau_{p}}{C_{n o}} ; \quad R_{n}=\frac{\tau_{n}}{C_{n o}} ; \quad R_{n t}=\frac{\tau_{n t}}{C_{n o}} ; R_{n r}=\frac{\tau_{n r}}{C_{n o}} ; \\
I_{i} & =\frac{V_{i}}{R_{n t}} ; \quad I_{a}=C_{n o} V_{i}\left(v_{n} \zeta_{n}+v_{p} \zeta_{p}\right)
\end{aligned}
$$

The diffusion currents can be found solving the continuity equations for the steady state and applying the appropriate boundary conditions [6]. The steady-state diffusion currents then are given by

$$
\begin{aligned}
I_{n} & =\frac{V_{n}}{R_{n d}}+\beta_{n} P_{i n}-I_{d n} \\
I_{p} & =\frac{V_{p}}{R_{p d}}+\beta_{p} P_{i n}-I_{d p}
\end{aligned}
$$

The current through the photodetector is the sum of the hole diffusion current in the n-region, the current through the i-region, that includes the electron diffusion current in the p-region, the displacement current and tunnelling and parasitic currents

$$
I_{J}=I_{p}+I_{i}+C_{T} \frac{\mathrm{d} V_{J}}{\mathrm{~d} t}+I_{p a r}
$$

where $V_{J}$ is the junction voltage, $C_{T}=C_{s}+C_{J}, C_{s}$ is the parasitic capacitance and $C_{J}$ is the junction capacitance and $I_{\text {par }}$ represents the tunnelling and parasitic current contributions.

\section{IMPLEMENTATION OF THE LARGE-SIGNAL PIN MODEL}

The implementation of the above model has been pursued with the help of the so called symbolic defined device (SDD), which is able to model linear and nonlinear devices and can be used in harmonic-balance simulations. The SDD model is defined through relations for the terminal currents and voltages. The major task here is the appropriate formulation of the individual elements of the equivalent circuits in order to achieve convergence. The implemented SDD in Agilent ADS2003C is provided in fig.3. The model includes bandgap engineering and

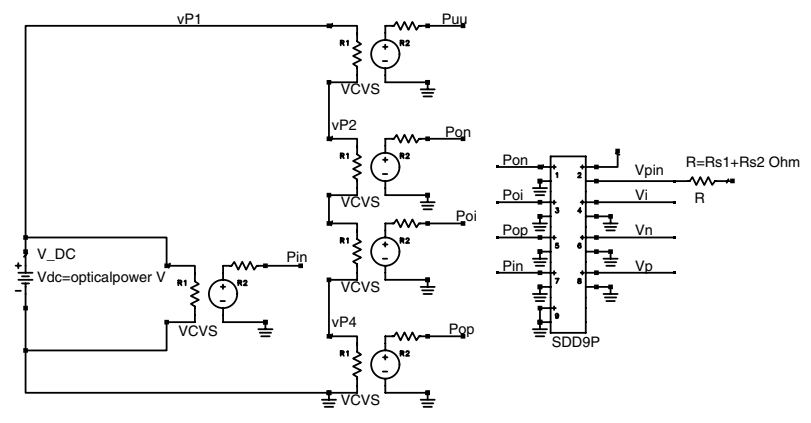

Fig. 3. Equivalent circuit for light input

is currently being extended to super-lattice structures. The possibility of built-in fields is also considered as 
a function of the bandgap in the individual layers. The full model is capable of simulations of travelling-wave structures. It employs a coupled line model for the electrical signal and an optical waveguide. The travelling-wave model will be subject of another paper.

\section{RESULTS}

This circuit has been verified with data available for an APD and an ultra-fast photodetector. The results for the DC characteristics for two diodes are provided in fig. 5. In fig. 5a) a comparison between measured and simulated results is shown for an avalanche photo diode (APD) as reported in [6]. The other diode in fig. 5b) has been developed at the University of Erlangen [] and is part of a super-lattice nipnip $\mathrm{THz}$ photo-diode, which utilizes several cascaded PIN integrated diodes together with the according recombination diodes. The DC photocurrent are according to the expected characterstics. The performance

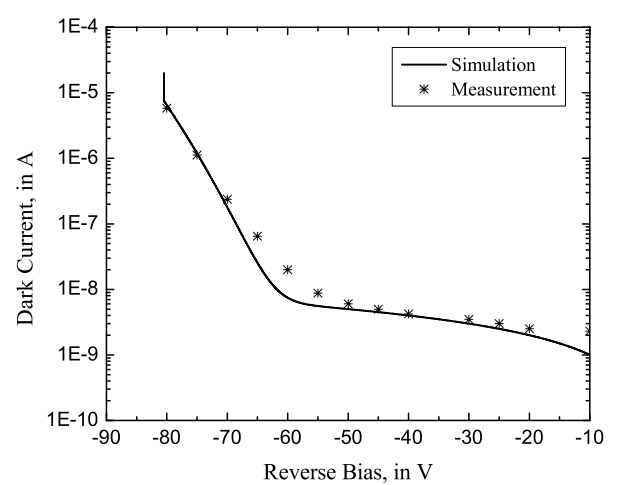

a)

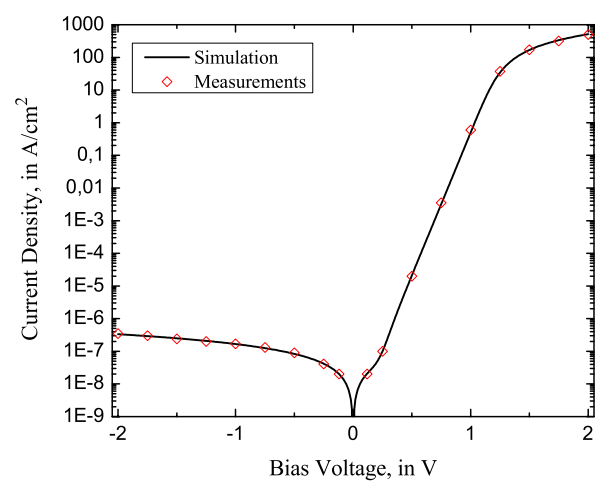

b)

Fig. 4. Comparison of simulated and measured I/V characteristics of an APD diode [6] and an ultra-fast PIN diode fabricated by the Univ. of Erlangen [7].

of the diodes of fig. 5 under illumination is illustrated in fig. 4. The DC photocurrent is shown in this figure for a number of optical powers. In the case of the APD diode only the reverse characteristics are shown in the figure.

Large-signal harmonic-balance analysis can then be used for the determination of the embedding impedances for ultra-fast photodiodes. The photodetector has been simulated in a setup with two laser colors, resulting in a

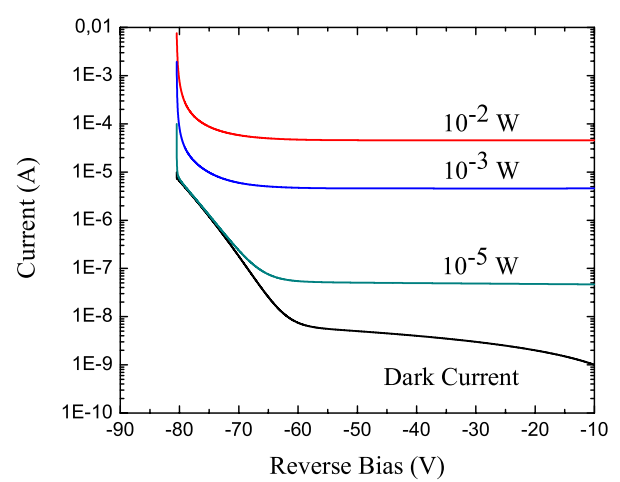

a)

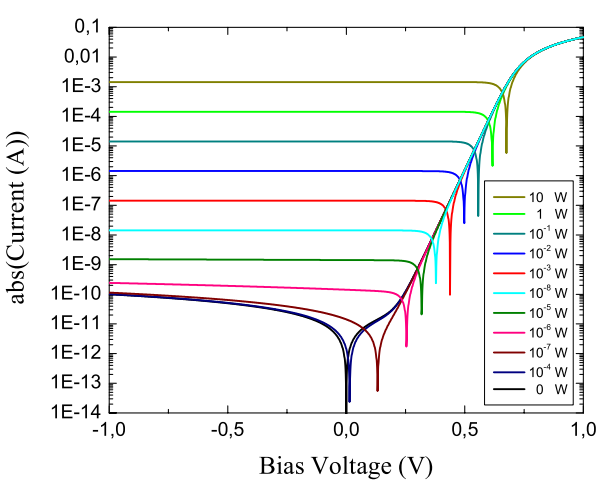

b)

Fig. 5. Simulated I/V characteristics of an APD diode [6] and an ultrafast PIN diode fabricated by the Univ. of Erlangen [7] under illumination with different optical power levels.

beat frequency, which has been varied from $100 \mathrm{GHz}$ up to $1000 \mathrm{GHz}$. The output power versus the beat frequency for the ultra-fast PIN diode from fig. 5 is provided in fig. 6 together with the embedding impedances. It can be verified that the output power drops with frequency according to $1 / f^{4}$ which indicates that the diode suffers from transit time and $R C$ time constant limitations.

The real part of the impedance is relatively low and equal to $R_{P I N} \approx 13 \Omega$. This indicates that relatively low impedance embedding circuits or antennas have to be used in order to match the diode.

\section{Conclusions}

The paper presents a large-signal model for PIN diodes applicable up to $\mathrm{THz}$ frequencies. Comparison between measured and simulated DC and AC characteristics show excellent agreement. The model can also be used for travelling-wave photodiodes and the implementation of the travelling-wave structure into the CAD environment is currently under way and will be shown during the conference.

\section{REFERENCES}

[1] A. Malcoci, A. Stöhr, R. Heinzelmann, K. Hagedorn, R. Güsten, F. Schäfer, H. Stüer, F. Siebe, P. van der Wal, V. Krozer, M. Feiginov, and D. Jäger, "Photonic (sub)millimeterwave local oscillators," in Microwaves, Radar and Wireless Communications, 2002. 


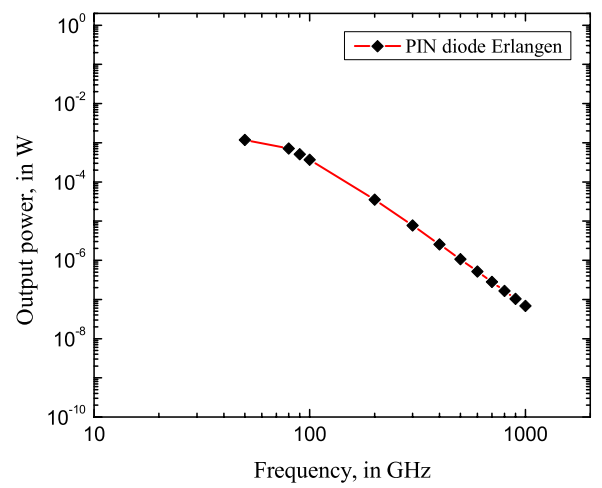

a)

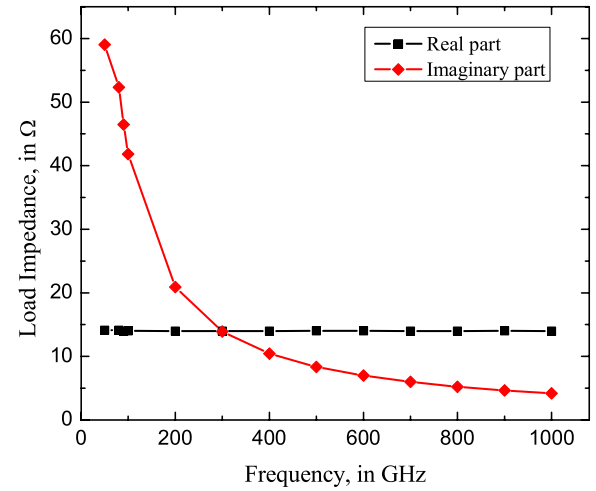

b)

Fig. 6. Comparison of simulated and measured I/V characteristics of an APD diode [6] and an ultra-fast PIN diode fabricated by the Univ. of Erlangen [7].

MIKON-2002. 14th International Conference on. 2002, vol. 3, pp. 722-734 vol.3, Telecommun. Res. Inst.

[2] M. Dentan and B. de Cremoux, "Numerical Simulation of the Nonlinear Response of a p-i-n Photodiode Under High Illumination," Journal of Lightwave Technology, vol. 8, no. 8, pp. 1137-1144, 1990.

[3] J. J. Jou, C.-K. Liu, C.-M. Hsiao, H.-H. Lin, and H.-C. Lee, "TimeDelay Circuit Model of High-Speed p-i-n Photodiodes," IEEE Photonics Technology Letters, vol. 14, no. 22, pp. 525-5274, 2002.

[4] G. Torrese, I. Huynen, M. Serres, D. Gallagher, M. Banham, and A. Vander Vorst, "An Analytical Small-Signal Bias-Dependent Nonuniform Model for p-i-n Traveling-Wave Photodetectors," IEEE Trans. Microwave Theory \& Techniques, vol. MTT-50, no. 11, pp. 2553-2557, 2002.

[5] G. George and J. P. Krusius, "Transient Response of HighSpeed p-i-n Photodiodes Including Diffusion Effects," Solid-State Electronics, vol. 37, no. 11, pp. 1841-1847, 1994.

[6] W. Chen and S. Liu, "PIN Avalanche Photodiodes Model for Circuit Simulation," vol. QE-32, no. 8, pp. 2105-2111, 1996.

[7] O. Klar M.Eckhardt A. Schwanhöußer S. Malzer D. Driscoll M. Hanson A. C. Gossard G. Loata T. Löffer H. Roskos G. H. D öhler, F. Renner, "THz-Photomixer Based on Quasi-Ballistic Transport,"

J. of Semiconductor Science and Technology, 2005. 